\title{
Bayesian Credible Interval Finite Element Method Applied in the Bird-Striking Canopy Problem
}

\author{
Changhong Liu, ${ }^{1, a}$, Bo Liu, Cunpan Yang, Junhong Qi, Yanbo Deng \\ (Key Lab of Safety Science of Pressurized System, Ministry of Education School of Engineering of \\ Science and Technology, East China University of Science and Technology, China, 200237) \\ aliuch1957@qq.com
}

Keywords: Bayesian theory; Prior information; Posterior distribution; Bayesian credible interval Abstract: For the uncertainty problem of structure strength of bird-striking canopy, firstly, the random finite element model is established by the prior random information. Secondly, according to the real test data, the Bayesian posterior distribution of the structure strength can be determined and the mean value and variance of the distribution without prior information also can be obtained, which can determine their bird-striking credible intervals of Bayesian theory. Finally, the example illustrates the effectiveness of the proposed method.

\section{Introduction}

For practical engineering problems, the random phenomena in structure analysis are very important [1], so the stochastic finite element method is widely applied in the field. According to the theory of probability statistics, the random parameters in a stochastic model need to be determined by a large number of experimental data. If the test data is not sufficient, it cannot be accurately determined by the traditional methods of probability statistics. According to Bayesian probability statistical methods, the prior information can be obtained from the data of the previous analogous test, and the posterior information can be obtained with the use of a small amount of actual information based on recent experiences, so such problems of the small data can be resolved and Bayesian probability statistics method has been widely used ${ }^{[1-4]}$. A new method in the stochastic finite element method based on Bayesian confidence interval is proposed.

\section{Stochastic finite element method in Bayesian credible interval}

Assuming that $x$ are random variables in the stochastic finite element model and $x_{m u}$ are the mean values, when the mean values of the above parameters are brought into the finite element model, the finite element model of the corresponding stochastic parameters can be obtained ${ }^{[5]}$ from the following Eq.1.

$$
\left[K_{m u}\right]\left\{U_{m u}\right\}=\left\{P_{m u}\right\}
$$

While, $\left[K_{m u}\right]$ is the stiffness matrix, and $\left\{U_{m u}\right\}$ is the displacement vector, $\left\{P_{m u}\right\}$ is the load vector.

According to the traditional finite element method of the design and calculation, it can be concluded that the finite element results by the mean values of parameters $\left\{x_{m u}\right\}$ are the mean values of the results in the stochastic model.

The mean value of the random variable of the finite element model of the structure is the Eq.2.

$$
\theta=Z_{m u}
$$

Based on Bayesian theory, a Bayesian function of the posterior normal distribution with the unknown variance and known mean value will be gained when we assumed that the mean values of the parameters are known and the standard deviations are unknown, and the result is the random variable in the normal distribution. Then we can take the Bayesian credible interval $\left[Z_{L}, Z_{R}\right]$ as the interval of the parameter with unknown posterior variance.

According to Bayesian theory, when the mean value $\theta$ is known, assuming that $Y=\left(Y_{1}, \ldots, Y_{n}\right)$ is 
obtained from the real data which obeys the normal distribution $N\left(\theta, \sigma^{2}\right)$, and the prior distribution of $\sigma^{2}$ is without prior information, then the process of solving the posterior distribution is as follows : Assuming $S_{*}^{2}=\frac{1}{n} \sum_{k=1}^{n}\left(Y_{k}-\theta\right)$, the likelihood function of $\sigma^{2}$ (or the probabilistic density function of $S^{2}$ ) in the normal distribution $N\left(\theta, \sigma^{2}\right)$ can be expressed as the following Eq.3.

$$
l\left(\sigma^{2} \mid t\right)=\frac{\left(n / \sigma^{2}\right)^{n / 2}}{2^{n / 2} \Gamma(n / 2)}\left(S_{*}^{2}\right)^{n / 2} \exp \left\{-\frac{n S_{*}^{2}}{2 \sigma^{2}}\right\}
$$

And the posterior distribution function of $\sigma^{2}$ obeys the inverse-Gama distribution: $\Gamma^{-1}\left(n / 2, n S_{*}^{2} / 2\right)$, which is equivalent to the following Eq.4.

$$
\pi\left(\sigma^{2} \mid S_{*}^{2}\right)=\frac{\left(n S_{*}^{2} / 2\right)^{n / 2}}{\Gamma(n / 2)}\left(\sigma^{2}\right)^{-(\mathrm{n} / 2+1)} \exp \left\{-\frac{n S_{*}^{2}}{2 \sigma^{2}}\right\},\left(\sigma^{2}>0\right)
$$

So the mean value and variance of the posterior distribution of $\sigma^{2}$ are the Eq.5.

$$
E\left(\sigma^{2}\right)=\frac{\lambda}{r-1}, \quad D\left(\sigma^{2}\right)=\frac{\lambda^{2}}{(\mathrm{r}-1)^{2}(\mathrm{r}-2)}, \quad\left(\mathrm{r}=\frac{n}{2}, \lambda=\frac{n S_{*}^{2}}{2}\right)
$$

At the same time, the definition of the credible interval stipulates as follow: if the posterior distribution of $\theta$ is $\pi(\theta \mid \mathrm{x})$, for the given sample $x$ and probability $(1-\alpha)$, the existence of the upper credible limit $\theta_{U}$ and the lower credible limit $\theta_{L}$ of Bayesian credible $(1-\alpha)$ level satisfy the following Eq.6.

$$
P\left(\theta_{L} \leq \theta \leq \theta_{U}(x) \mid x\right)=1-\alpha
$$

Then, $\left[\theta_{L}, \theta_{U}\right]$ can be called as the Bayesian credible interval of the credible level $(1-\alpha)$. While, when the upper credible limit $\theta_{U}$ satisfies the following Eq.7

$$
P\left(\theta \leq\left.\theta_{U}(x)\right|_{X}\right) \geq 1-\alpha
$$

It will be called as the single side interval of the upper credible level.

According to the principle of reliability design, the posterior distribution of the variance is the following equation Eq.8.

$$
\sigma^{2^{\prime}}=E\left(\sigma^{2}\right)+\xi D\left(\sigma^{2}\right), \quad \xi \geq 1
$$

Therefore, the Bayesian credible interval of the credible level $(1-\alpha)$ is defined as the following Eq.9, when $S=\sqrt{\sigma^{2 \prime}}$ is assumed.

$$
Z_{\mathrm{PINT}}=Z_{\mathrm{mu}} \pm S, \quad \text { or }\left[Z_{\mathrm{PINT}}, Z_{\mathrm{PINU}}\right]=\left[\left(Z_{\mathrm{mu}}-S\right),\left(Z_{\mathrm{mu}}+S\right)\right]
$$

While, $\mathrm{Z}_{\mathrm{PINT}}$ is the posterior credible interval expectation of the random finite element results (such as the displacement or stress).

\section{Example}

A bird-striking canopy problem is considered with the SPH and FEM coupling method, and the bird-impacting canopy model ${ }^{[5]}$ is shown in the Fig. 1 . The shell element is used for the skin and the supporting member, and the rivet is simplified as a beam element. The elastic-plastic material is used 
to simulate the constitutive relation of the aluminous canopy. And a bird's body model is established by the SPH (Smoothed Particle Hydrodynamics).

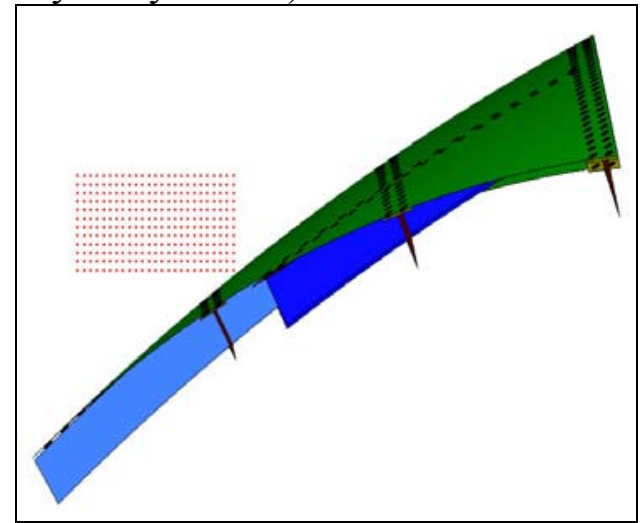

Fig.1 A model of bird striking on the canopy

Using the method proposed in this paper, the mean value of the maximum stress in the random model is $284 \mathrm{MPa}$, which is less than the material's design strength $350 \mathrm{MPa}$. According to the above method, the data of 10 cases of bird-striking accidents can be calculated as follows: $S_{*}{ }^{2}=40000$ $(M P a)^{2}, \quad l=200000(M P a)^{2}, r=2.5$. So the mean value and variance of $\sigma^{2}$ are as the following Eq.10.

$$
E\left(\sigma^{2}\right)=22222.22 \mathrm{MPa}^{2}, \quad D\left(\sigma^{2}\right)=7856.74^{2} \mathrm{MPa}^{2}
$$

When we take the standard derivation as $\Delta=\sqrt{22222.22+7856.74}=173 \mathrm{MPa}$, considering random factor, the maximal stress in the bird striking will be $(284+173)=457 \mathrm{MPa}$.

As the value is absolutely larger than the material's design strength, so the canopy structure must be redesigned.

\section{Conclusion}

In this paper, a Bayesian interval finite element method is proposed, the main steps are following:

(1) Firstly, by referring to the relevant prior information and experience, the mean value of the random parameter's posterior distribution can be determined by the recent real test data.

(2) Then according to the Bayesian theory, the response of the stochastic finite element model can be obtained, which satisfies the normal distribution with the unknown variance and known mean value;

(3) The posterior distribution of the variance can be obtained from the real information when the prior distribution of the variance is unknown;

(4) Finally, a Bayesian credible interval can be obtained by the response of the stochastic finite element model.

The example shows that this method took into account the random parameter distribution of the similar problem. Therefore, the method proposed in this paper will be more realistic.

\section{References}

[1] L.S. Wei, W.P. Zhang, in: Bayesian analyses, Hefei, USTC (2013) (In Chinese).

[2] M.S. Hamads, A.G. Wilson, C. Shane Reese and Harry F. Marts, in: Bayes reliability, New York, Springer (2008).

[3] A. Baklizi: Journal of Statistical Computation and Simulation Vol. 84 (2014), p.2670.

[4] C.H. Liu, C. Qiu: Journal of Applied mathematics and mechanics Vol. 26 (2005), p.1312 (In Chinese).

[5] C.H. Liu, J. Luo: Journal of Guangxi University Vol.37 (2012), p.899 (In Chinese). 